\title{
Energy analysis of holographic lenses for solar concentration
}

\author{
Julia Marín-Sáez ${ }^{\mathrm{a}}$, M. Victoria Collados ${ }^{\mathrm{b}}$, Daniel Chemisana ${ }^{\mathrm{a}}$, Jesús Atencia*b \\ ${ }^{a}$ Applied Physics Section of the Environmental Science Department, Polytechnic School, University \\ of Lleida, E-25001 Lleida, Spain; \\ ${ }^{\mathrm{b}}$ Applied Physics Department, Aragon Institute of Engineering Research (I3A), University of \\ Zaragoza, E-50009 Zaragoza, Spain
}

\begin{abstract}
The use of volume and phase holographic elements in the design of photovoltaic solar concentrators has become very popular as an alternative solution to refractive systems, due to their high efficiency, low cost and possibilities of building integration. Angular and chromatic selectivity of volume holograms can affect their behavior as solar concentrators. In holographic lenses, angular and chromatic selectivity varies along the lens plane. Besides, considering that the holographic materials are not sensitive to the wavelengths for which the solar cells are most efficient, the reconstruction wavelength is usually different from the recording one. As a consequence, not all points of the lens work at Bragg condition for a defined incident direction or wavelength. A software tool that calculates the direction and efficiency of solar rays at the output of a volume holographic element has been developed in this study. It allows the analysis of the total energy that reaches the solar cell, taking into account the sun movement, the solar spectrum and the sensitivity of the solar cell. The dependence of the recording wavelength on the collected energy is studied with this software. As the recording angle is different along a holographic lens, some zones of the lens could not act as a volume hologram. The efficiency at the transition zones between volume and thin behavior in lenses recorded in Bayfol HX is experimentally analyzed in order to decide if the energy of generated higher diffraction orders has to be included in the simulation.
\end{abstract}

Keywords: Holographic lenses, solar concentration, volume holograms, photovoltaics

\section{INTRODUCTION}

Holographic Optical Elements (HOEs) are a versatile technology that has multiple applications. One of them is solar concentration, for which different configurations have been studied ${ }^{1}$. With the adequate design, both holographic gratings and cylindrical and spherical lenses can act as solar concentrators.

The efficiency of HOEs reaches its maximal values for certain conditions depending, among other parameters, on the incidence angle and wavelength, that is, they present angular and chromatic selectivity.

HOEs can be designed so that their efficiency reaches high values for a particular wavelength range, which in solar concentration applications needs to take into account the incident solar spectrum and also the kind of receiver the solar radiation will be directed to. Figure 1 shows the ASTM standard solar spectrum AM 1.5D, normalized to $1000 \mathrm{~W} / \mathrm{m}^{2}$ over the wavelength range 350-1200 nm, depicted with a blue line and left $y$-axis. The spectral response curve of a mono-crystalline Si photovoltaic cell is plotted as well, with a red line and right $y$-axis. The spectral range a HOE should diffract more efficiently towards this type of PV cell may be determined with a compromise between these two curves, and would be then centered at $800 \mathrm{~nm}$, which will be referred to as the target wavelength. 


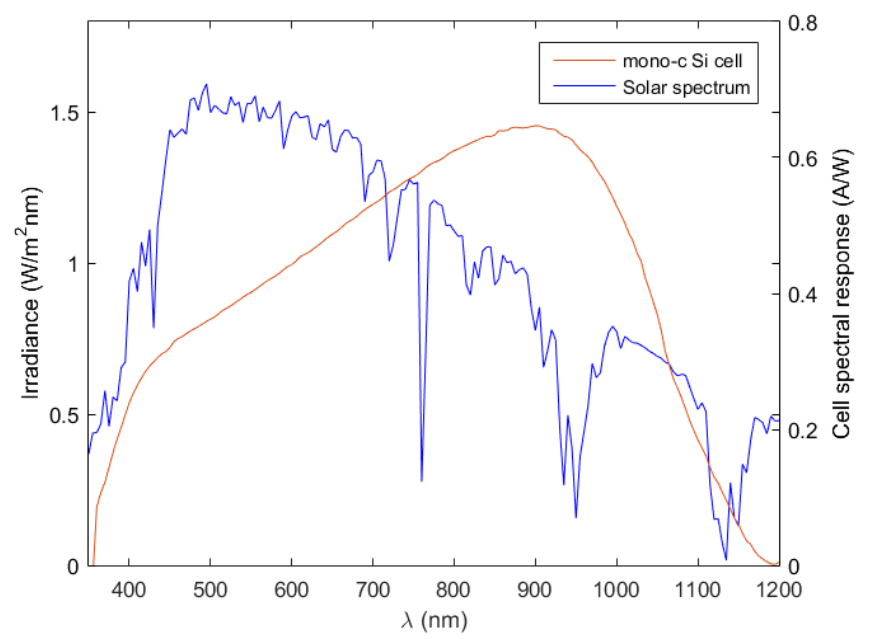

Figure 1. ASTM standard solar spectrum AM 1.5D, normalized to $1000 \mathrm{~W} / \mathrm{m}^{2}$ over the wavelength range $350-1200 \mathrm{~nm}$ (blue curve, left $y$-axis). Cell response of a mono-crystalline Si cell (red curve, right $y$-axis).

Regarding the angular selectivity, since the apparent position of the Sun in the sky changes along the day and the year, tracking of its movement may be required in order to avoid the efficiency loss that occurs when the incident angle is too different from the optimal conditions. When the incidence angle varies along one direction, that lies on the plane defined by the wavevectors of the recording beams, the efficiency decreases promptly (direction of high angular selectivity); whereas if it varies along the perpendicular direction, the efficiency decreases slowly (direction of low angular selectivity). Cylindrical holographic lenses are a rather suitable option for solar concentration, because if they are placed in such a way that the direction of low angular selectivity matches the daily solar movement direction ${ }^{2,3}$, tracking is only needed in one axis, simplifying the final system design.

Volume HOEs can achieve 100\% efficiency, which is desired in most applications. However, their chromatic and angular selectivity are also very high. HOEs operating at the limit of the volume regime or even in the transition regime achieve very high efficiency as well, whereas enlarging the acceptance angular and spectral range. Holographic cylindrical lenses for solar concentration may benefit from that, since a broad spectrum is desired to be diffracted.

Among the different photosensitive materials for holographic recording, photopolymers have been frequently used for the recording of holographic lenses ${ }^{4-6}$ or gratings ${ }^{7}$. Bayfol HX, a photopolymeric recording material developed by Covestro $\mathrm{AG}^{8}$, offers very interesting advantages, such as the lack of requirement of a wet processing after the recording; a simple photocuring process is needed instead. It can also provide the dynamic range necessary for recording HOEs that are highly efficient at $800 \mathrm{~nm}^{9}$.

Holographic cylindrical lenses have been studied in certain systems designed to concentrate solar irradiance onto one or more photovoltaic cells. Gordon et al.'s design ${ }^{10}$ is based on a system formed by two different holographic cylindrical lenses, each diffracting more efficiently a spectral range towards a different PV cell, so a broader part of the incident spectrum can be used. Ludman's configuration ${ }^{11,12}$ also includes several PV cells but its geometry presents an important characteristic: the transmitted order (which includes the infrared that may cause overheating of the PV cell and a worsening of its performance) does not reach any PV cell, thus avoiding this problem. With that scope in mind, some concentrating holographic systems have been proposed by the authors ${ }^{2,13}$, comprising volume cylindrical holographic lenses.

This work studies the effect of two different aspects of the design of cylindrical holographic lenses on the overall system performance and the collected irradiance on a PV cell. Section 2 covers a brief theoretical background on holographic recording, concentration coefficients used on solar applications and the developed software tool used for the theoretical calculations. The influence of the recording wavelength on the behavior of a holographic cylindrical lens is analyzed in section 3. Section 4 presents a preliminary analysis of a holographic cylindrical lens operating in the transition regime for a reconstruction wavelength of $800 \mathrm{~nm}$. 


\section{THEORETICAL BACKGROUND}

\subsection{Basis of holographic recording}

Holograms are recorded on a photosensitive medium with the interference of two coherent waves. A cylindrical holographic lens can be recorded with a cylindrical wave and a plane wave, with wave propagation vectors $\overrightarrow{k_{c}}$ and $\overrightarrow{k_{p}}$, respectively. The incidence angle of the cylindrical beam at the medium's surface is different along a direction, thus $\overrightarrow{k_{c}}=\overrightarrow{k_{c}}(\vec{r})$, which can be set to match the $y$-axis. Therefore, the interference pattern of the recording waves has a different frequency along $y$-direction, whereas in a plane grating this frequency is constant, since the angle between the recording beams does not vary. The grating vector, defined with equation (1), has a different direction and modulus at each point of the $y$-axis of a cylindrical holographic lens.

$$
\vec{K}=\overrightarrow{k_{p}}-\overrightarrow{k_{c}}
$$

The modulus of the grating vector is given by

$$
K=\frac{2 \pi}{\Lambda}
$$

where $\Lambda$ is the period of the interference fringes of the recording waves. In phase holograms, these variations of the intensity along the photosensitive material may be recorded on it by means of variations of the refractive index, so it can be written as

$$
n=n_{0}+n_{1} \sin (\vec{K} \cdot \vec{r})
$$

where $n_{0}$ is the average refractive index and $n_{1}$ is the index modulation. Since the grating vector $\vec{K}$ is different along $y$-direction, the period and frequency of the variations of the refractive index are different as well.

After the refractive index variations have been made permanent in the photosensitive medium, if the resulting hologram is a volume HOE, when it is illuminated two diffraction orders emerge at the output: the transmitted order (or 0 order of diffraction) and the first diffracted order (or +1 order). The efficiency of the diffracted order is maximal (and the one of the transmitted order minimal) when Bragg condition is met, defined with equation (4), where $\theta_{1}$ and $\theta_{2}$ are the angles of incidence of the transmitted and the diffracted wave.

$$
2 n_{0} \Lambda \sin \left(\frac{\theta_{1}-\theta_{2}}{2}\right)=\lambda
$$

If the hologram is not a volume one, more diffraction orders emerge, and the efficiency of the +1 diffracted order is consequently lower than in the previous case.

\subsection{Concentration coefficients for solar applications}

In solar concentration applications, a parameter called concentration coefficient is defined ${ }^{3}$, with equation (5):

$$
C C=\frac{\Phi_{C}}{\Phi_{L}} \frac{A_{L}}{A_{C}}=\frac{\sum_{\lambda} \Phi_{C, \lambda} \Delta \lambda}{\sum_{\lambda} \Phi_{L, \lambda} \Delta \lambda} \frac{A_{L}}{A_{C}}
$$

It is the ratio of the irradiance received by the photovoltaic cell (which is the quotient between the optical flux received, $\Phi_{C}$, and the surface of the cell, $A_{C}$ ) and the irradiance at the entrance pupil of the system (the quotient between the incident optical flux, $\Phi_{L}$, and the surface of the entrance pupil, that is, the surface of the lens $A_{L}$ ). The flux can also be 
expressed as the sum of the spectral flux corresponding to each wavelength, multiplied by the wavelength step ( $\sum_{\lambda} \Phi_{C, \lambda} \Delta \lambda$ for the flux on the cell and $\sum_{\lambda} \Phi_{L, \lambda} \Delta \lambda$ at the entrance pupil of the system).

For solar concentration purposes, it is necessary not only to calculate the concentration coefficient defined in equation (5) as the ratio of irradiances, but also the ratio of current intensities generated by the PV cell with and without the holographic concentrator, in order to take into account the spectral response of the cell. It is defined in equation (6), where $S R_{\lambda}$ is the spectral response of the cell (the current intensity generated due to the optical flux that reaches the cell).

$$
C C_{\text {currentintensity }}=\frac{\sum_{\lambda} \Phi_{C, \lambda} S R_{\lambda} \Delta \lambda}{\sum_{\lambda} \Phi_{L, \lambda} S R_{\lambda} \Delta \lambda} \frac{A_{L}}{A_{C}}
$$

\subsection{Software tool}

A software tool has been developed in Matlab in order to study the performance of $\mathrm{HOEs}^{3,14}$. It is based on a ray tracing algorithm, so the behavior of each point of the HOE is examined independently. This is based on the assumption that it can be considered that for each point of a cylindrical holographic lens the recording waves are two plane waves, that is, each point of the HOE can be treated as a local grating.

The efficiency of each ray that reaches the HOE is calculated based on the coupled wave differential equations, either solving them applying Kogelnik's Coupled Wave Theory ${ }^{15}$ when it is a volume hologram, or solving them numerically when it is not ${ }^{16}$.

The algorithm takes into account Fresnel reflection $\operatorname{losses}^{17}$ at both the entrance and output interfaces, and the AM 1.5D solar spectrum can be considered to be the incident spectrum. Since sunlight is depolarized, the efficiencies are calculated for both polarizations and then averaged to obtain the final efficiency.

\section{ANALYSIS OF THE INFLUENCE OF THE RECORDING WAVELENGTH ON THE COLLECTED ENERGY}

Three recording wavelengths have been chosen for this analysis: two typical laser wavelengths (532 and $632.8 \mathrm{~nm}$ ) and the target wavelength $(800 \mathrm{~nm})$. One may assume beforehand that the best results would be achieved when the recording wavelength matches the target wavelength, since less aberration effects would appear at the reconstruction. However, the reconstructed image quality is not a critical factor in solar concentration applications. Instead, the total energy belonging to a certain spectral range that reaches the receiver is desired to be optimum, and it is not necessary that the rays converge to a point, but a small area.

The three lenses were designed so that Bragg condition would be met at the center of the lens when replaying with $\theta_{0}=0^{\circ}$ and $\lambda=800 \mathrm{~nm}$. The angle with $z$-axis (defined perpendicular to the HOE, so that the YZ-plane is the recording plane) of the diffracted wave at that point under those conditions is $\theta_{+1}=45^{\circ}$ for all three lenses, so the spatial period is the same at the center and varies similarly along the lens. The paraxial image at the reconstruction is located in the same Z-plane, $70 \mathrm{~mm}$ away from the HOE.

The angles of incidence of the two recording waves are presented in Table 1. They ensure that the whole HOEs operate at the volume regime when reconstructing with $\lambda=800 \mathrm{~nm}$.

A global analysis of the performance of each lens has been carried out by means of the software tool mentioned in section 2. The direction of the diffracted rays with incidence angle $\theta_{0}=0^{\circ}$ and wavelengths from 350 to $1200 \mathrm{~nm}$ was calculated, as well as their efficiency. As expected when the recording wavelength does not match the reconstruction wavelength, the efficiency of the $800 \mathrm{~nm}$ rays that impact the center of each lens is maximal, and it decreases when moving away towards positive or negative values of $y$, as shown in Figure 2. That is not the case of the lens recorded 
with $\lambda_{R}=800 \mathrm{~nm}$, where Bragg condition is met at every point of the lens when reconstructing with $\theta_{0}=0^{\circ}$ and $\lambda=800$ $\mathrm{nm}$. The efficiency of two other sample wavelengths near the target wavelength, 700 and $900 \mathrm{~nm}$, is also represented in Figure 2 for each lens. Since both polarizations are taken into account in the calculations and the efficiency of the 800 $\mathrm{nm}$ rays is $100 \%$ at the center for one of them, but not for the other one, the final efficiency does not reach $100 \%$ in any case.

Table 1. Angles of incidence in air $\theta_{p}$ (of the plane wave) and $\theta_{c}$ (of the cylindrical wave, at the center of the lens) of the recording waves of the considered holographic lenses.

\begin{tabular}{|c|c|c|c|}
\hline & $\lambda_{\mathbf{R}}=\mathbf{5 3 2} \mathbf{~ n m}$ & $\lambda_{R}=\mathbf{6 3 2 . 8} \mathbf{~ n m}$ & $\lambda_{\boldsymbol{R}}=\mathbf{8 0 0} \mathbf{~ n m}$ \\
\hline$\theta_{p}\left(^{\circ}\right)$ & 7.2 & 4.5 & 0 \\
\hline$\theta_{c}\left(^{\circ}\right)$ & 36.5 & 39.7 & 45 \\
\hline
\end{tabular}
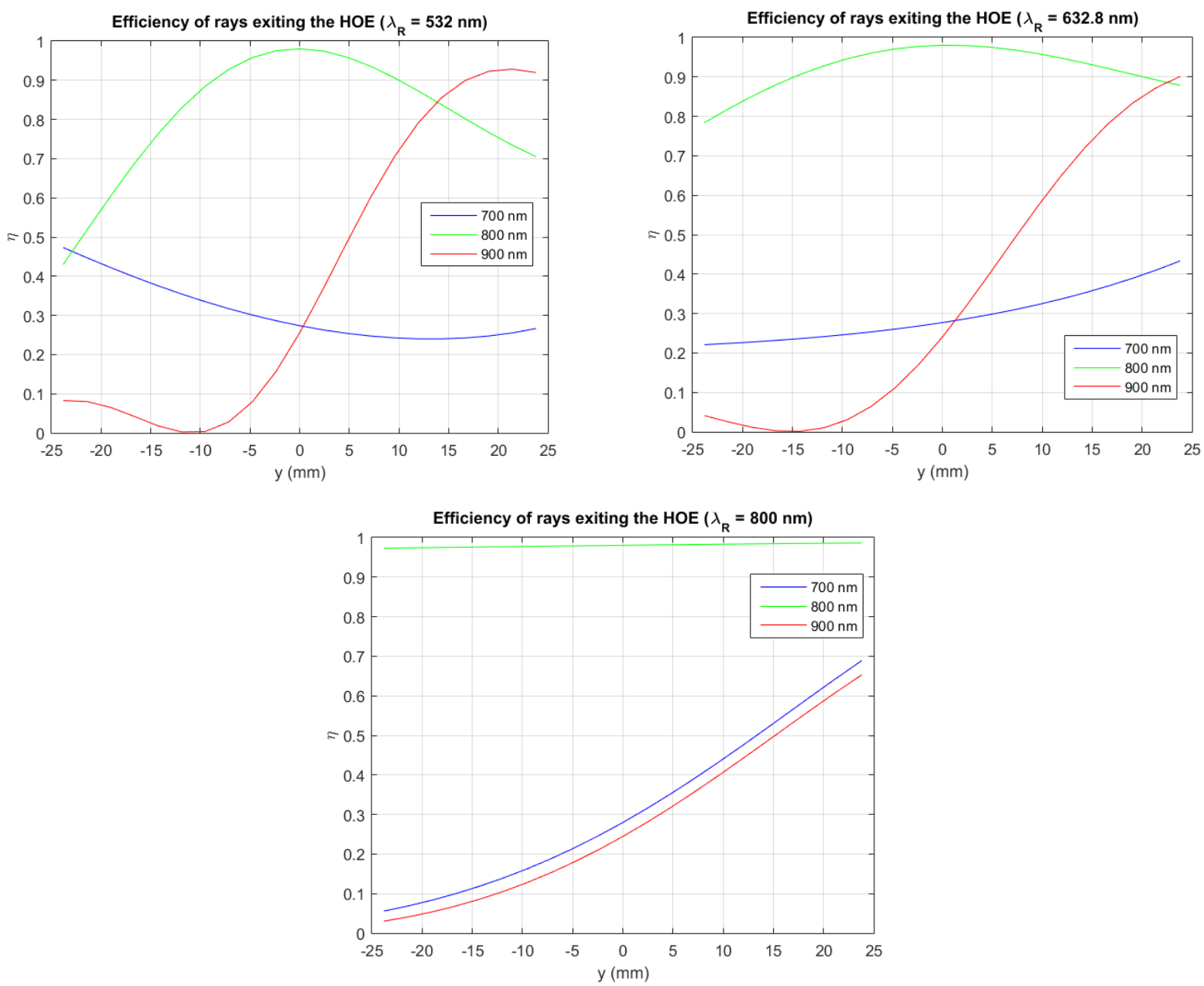

Figure 2. Efficiency of 700 (blue curves), 800 (green curves) and $900 \mathrm{~nm}$ (red curves) rays when reconstructing with $\theta=0^{\circ}$ at the output of lenses recorded at 532 (top left), 632.8 (top right) and $800 \mathrm{~nm}$ (bottom). 
Not only is the efficiency of each ray relevant for this analysis, but also its direction, to determine if they would be collected by the PV cell. The calculated ray tracing for those three wavelengths is plotted in Figure 3. The presence of aberrations in the $800 \mathrm{~nm}$ rays diffracted by the lenses recorded with 532 and $632.8 \mathrm{~nm}$ can be clearly noticed, as well as the lack of them in the lens recorded with $800 \mathrm{~nm}$. The black line at around $z=40 \mathrm{~mm}$ in the first two cases and $z=60$ $\mathrm{mm}$ in the third one represents the PV cell located at a position, in which all $800 \mathrm{~nm}$ rays are collected and the final concentration coefficient is optimized. The spectral irradiance received by the cell with each of the three lenses when considering the AM 1.5D solar spectrum normalized to $1000 \mathrm{~W} / \mathrm{m}^{2}$ over the range $350-1200 \mathrm{~nm}$ is shown in Figure 4 . Fresnel reflection losses ${ }^{17}$ on the entrance and output interfaces are also taken into account in the calculations.
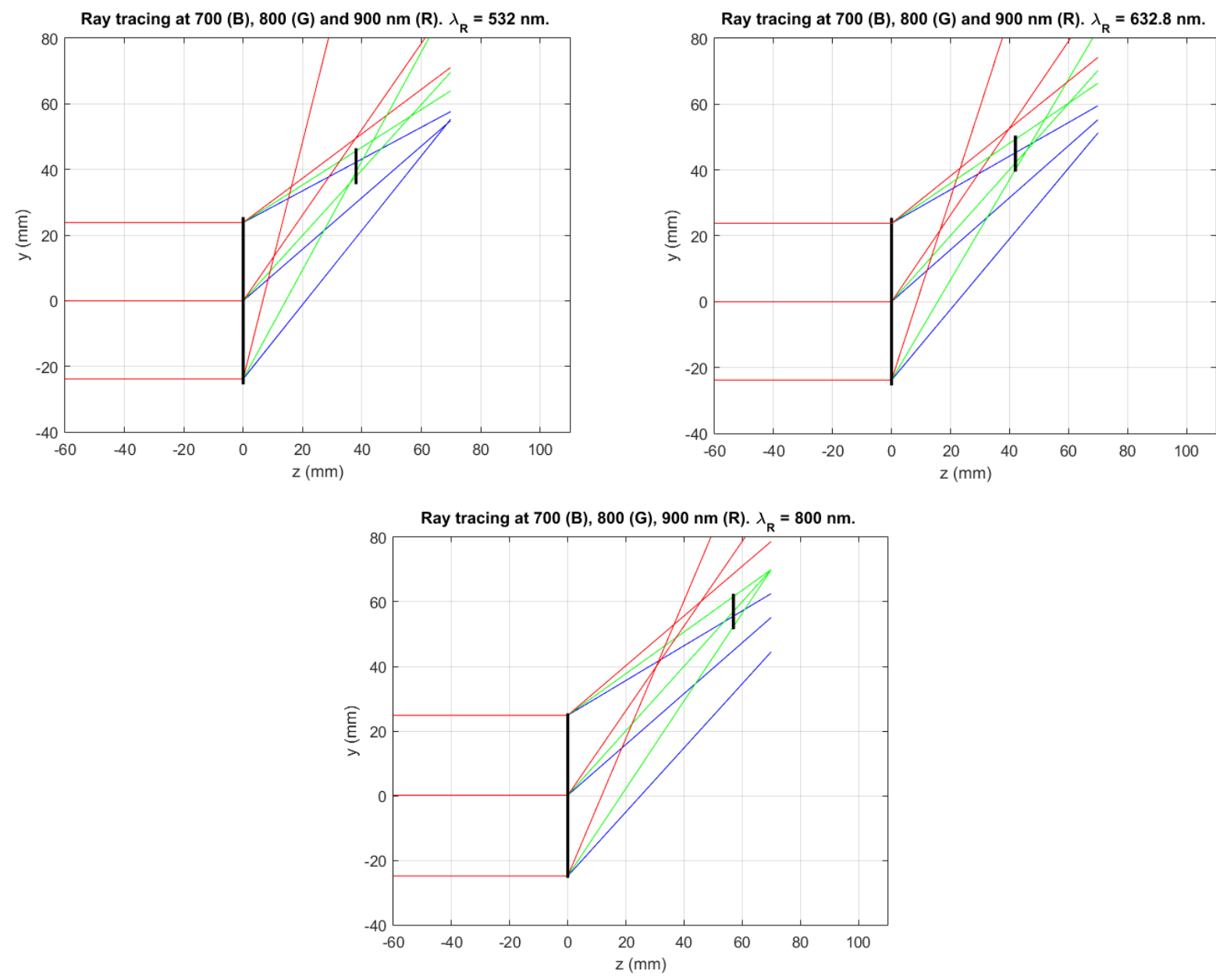

Figure 3. Ray tracing of rays of 700 (blue lines), 800 (green lines) and $900 \mathrm{~nm}$ (red lines) at the reconstruction stage of lenses recorded at 532 (top left), 632.8 (top right) and $800 \mathrm{~nm}$ (bottom), with $\theta=0^{\circ}$. The black lines around $z=40$ and 60 $\mathrm{mm}$ are possible placements of the PV cell.

The resulting optical and current intensity concentration coefficients, defined with equations (5) and (6), are presented in Table 2. They may seem low values, but it has to be taken into account that the concentrated spectrum belongs only to the wavelength range for which the PV cell has a spectral response, and no irradiance outside that wavelength range will cause an increase of the temperature of the PV cell, so its performance would not decline. Besides, more lenses could be included in the system to contribute with more irradiance corresponding to the diffracted or even the transmitted order to the same PV cell without an excessive increase of the temperature, such as the geometry described and analyzed in another work by the authors ${ }^{13}$. 


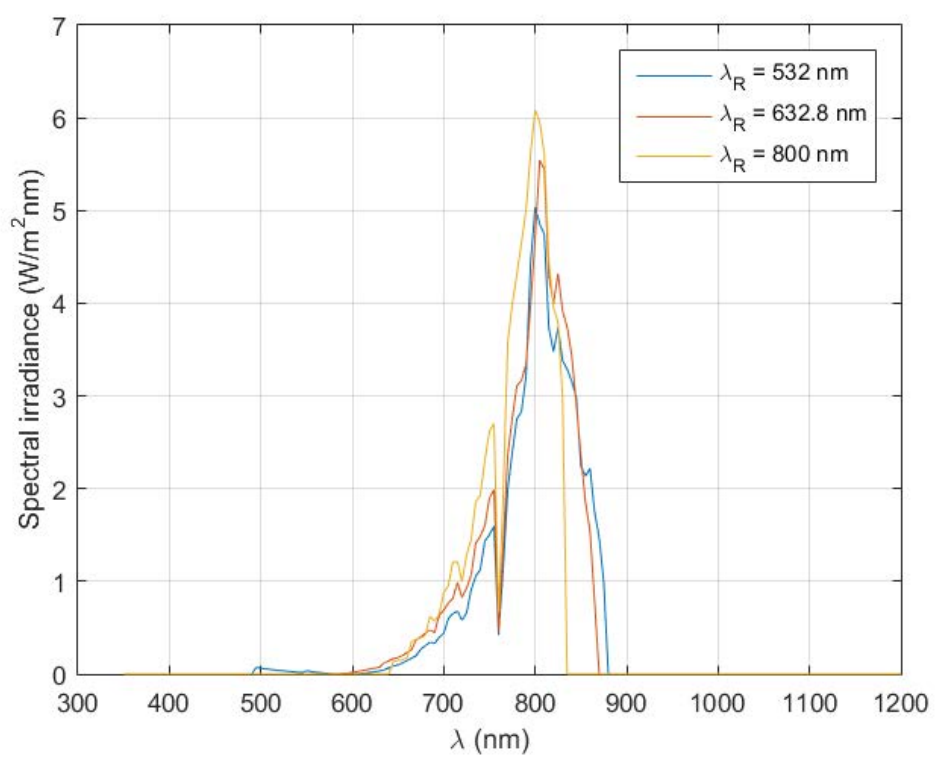

Figure 4. Spectral irradiance received by the PV cell when it is located at its optimum placement, with the lens recorded with $532 \mathrm{~nm}$ (blue curve), $632.8 \mathrm{~nm}$ (orange curve) and $800 \mathrm{~nm}$ (yellow curve). The entrance spectrum is the AM 1.5D solar spectrum normalized to $1000 \mathrm{~W} / \mathrm{m}^{2}$ over the range 350-1200 nm and Fresnel reflection losses at the entrance and output interfaces are considered.

Table 2. Concentration coefficients obtained with the optimum PV cell placement for each lens.

\begin{tabular}{|c|c|c|c|}
\hline & $\lambda_{R}=532 \mathbf{n m}$ & $\lambda_{R}=\mathbf{6 3 2 . 8} \mathbf{n m}$ & $\lambda_{R}=\mathbf{8 0 0} \mathbf{~ n m}$ \\
\hline$C C$ & 0.41 & 0.44 & 0.43 \\
\hline$C C_{\text {currentintensity }}$ & 0.57 & 0.60 & 0.57 \\
\hline
\end{tabular}

Recording the holographic cylindrical lens with the same wavelength as the target wavelength does not result in higher concentration coefficients than in the other cases. Furthermore, since no $800 \mathrm{~nm}$ photosensitive material adequate for this application has been found, it is not convenient to continue a thorough study with this recording wavelength. It is observed that the change in the concentration coefficients when recording a cylindrical lens with any of the other two wavelengths is not too big when the same design criterions are applied in order to obtain similar reconstruction conditions. Therefore, the election of the recording wavelength may be based on the laboratory equipment.

\section{HOLOGRAPHIC CYLINDRICAL LENSES OPERATING IN THE TRANSITION REGIME}

The high angular selectivity of holographic lenses operating in the volume regime may be a drawback in solar concentration applications, even if tracking in the direction of highest selectivity is carried out. Since the incidence angle of the cylindrical beam at the recording is different at each point of the lens along $y$-direction, the angle of incidence that fulfills Bragg condition at each point when reconstructing at $800 \mathrm{~nm}$ is also different from the one of the center of the lens. This difference is greater the further away from the center the considered point is. As a consequence, the efficiency of the $800 \mathrm{~nm}$ rays when reconstructing at Bragg angle for the center of the lens (which is, in principle, the reconstruction incidence direction that would be maintained with the tracking system) depends on the point of the lens 
that the rays impact, being maximum at the center and decreasing when moving away from it. This effect is emphasized the more into the volume regime the HOE operates at $800 \mathrm{~nm}$, since the angular selectivity is higher.

Therefore, holographic cylindrical lenses that operate at the limit of the volume regime or even at the transition regime between Bragg regime and Raman-Nath regime for the target wavelength and a small spectral range around it are of special interest for solar concentration applications. This way, a small deviation of the incident reconstruction direction from Bragg angle does not imply a big decrease of the efficiency.

The analytical solution of Kogelnik's Coupled Wave Theory can only be applied to volume HOEs, in which solely the transmitted and first order diffracted wave appear. In order to include higher diffraction orders, the coupled differential equations need to be solved numerically, which adds time to calculations, so it is not convenient unless it is necessary.

A holographic cylindrical lens has been recorded on Bayfol HX photopolymer at $532 \mathrm{~nm}$ with the recording geometry shown in Figure 5. The center of the lens fulfills Bragg condition when reconstructing with $\theta_{0}=0^{\circ}$ and $\lambda=800 \mathrm{~nm}$. Part of the HOE operates in the volume regime and part of it in the transition regime when reconstructing with this wavelength.

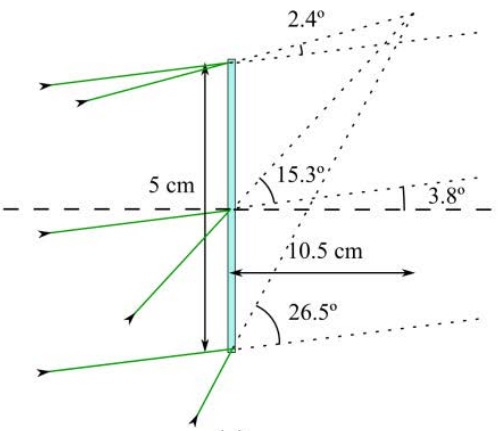

(a)

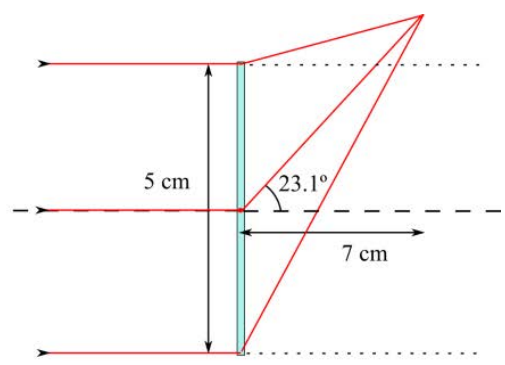

(b)

Figure 5. Recording geometry of the considered holographic cylindrical lens at $532 \mathrm{~nm}$ (left) and expected ray tracing at the reconstruction with $\theta=0^{\circ}$ and $800 \mathrm{~nm}$.

After the recording process, photocuring was carried out as previously described ${ }^{9}$. The intensity of each diffracted ray was then measured (with Newport Power Meter Model 1815-C with detector Model 818-SL, with an uncertainty of $5 \mu \mathrm{W}$ ) when illuminating a point of the HOE with a laser emitting at $800 \mathrm{~nm}$ (ThorLabs Laser Diode CPS808A, emitting $4.4 \mathrm{~mW}$ ) and at $\theta_{0}=0^{\circ}$. The relative efficiency of each order of diffraction at each point was calculated with equation (7):

$$
\eta_{i}=\frac{I_{i}}{\sum_{j} I_{j}}
$$

where $I_{i}$ is the measured intensity of the $i$-diffracted order. The resulting efficiencies are plotted in Figure 6 with filled circles. It can be noticed that for $y \leq-10 \mathrm{~mm}$ only the first diffracted order $(+1)$ and the transmitted order can be found, whereas when $y>-10 \mathrm{~mm}$ more diffraction orders emerge, achieving non negligible efficiencies. At the edge of the HOE where the angle between the recording beams was the smallest ( $y=+25 \mathrm{~mm}$ ), the efficiency of the -1 order of diffraction even reaches the value of the efficiency of the +1 order.

The efficiency of each diffraction order with $800 \mathrm{~nm}$ at the output of the lens has been calculated by a numerical resolution of the coupled differential equations. An estimated value of the index modulation, $n_{1}$, was assigned to each point of the lens according to its spatial frequency. The obtained theoretical efficiencies are shown in Figure 6 as well. Good agreement between theoretical and experimental results has been observed. 


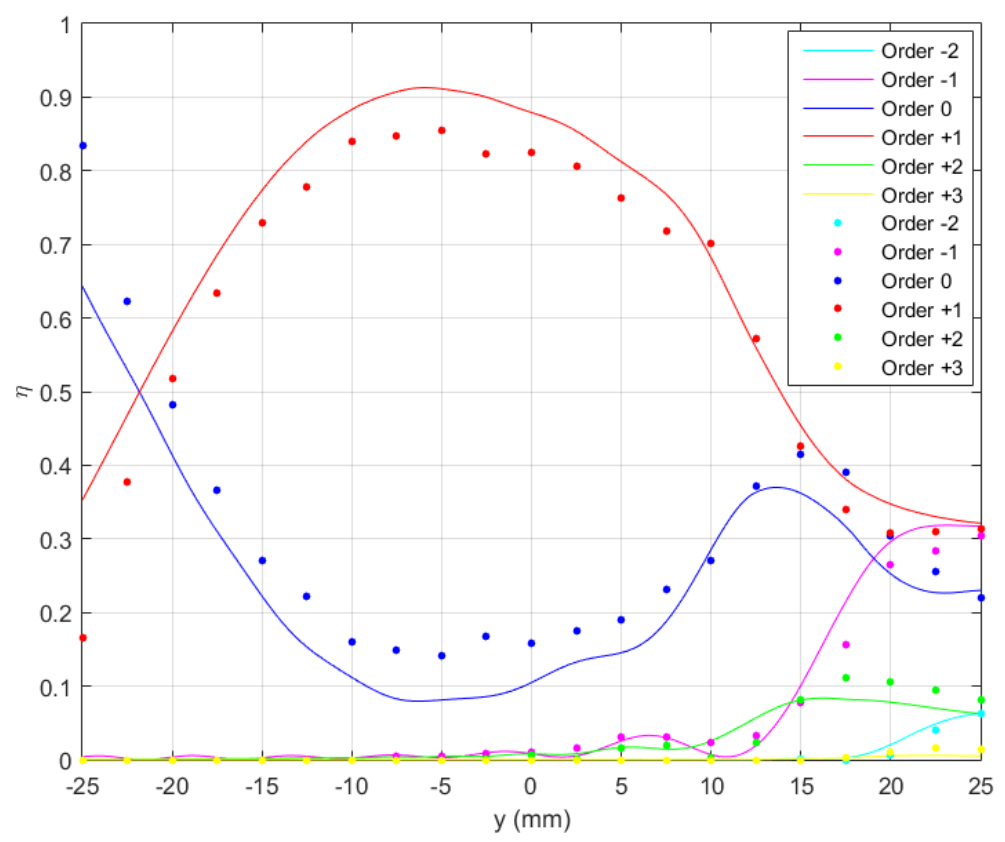

Figure 6. Experimental (filled circles) and theoretical (lines) efficiencies of every order of diffraction observed at each point of the considered holographic cylindrical lens when reconstructing with $\theta=0^{\circ}$ and $\lambda=800 \mathrm{~nm}$.

The direction of every ray was also calculated with the software. Figure 7 illustrates the ray tracing of all diffraction orders observed $(-2,-1,0,+1,+2$ and +3 orders, depicted with cyan $(C)$, magenta $(M)$, blue (B), red (R), green (G) and yellow $(\mathrm{Y})$ lines, respectively) that emerge from the center and the edges of the lens when reconstruction is carried out at $800 \mathrm{~nm}$ and $\theta_{0}=0^{\circ}$.

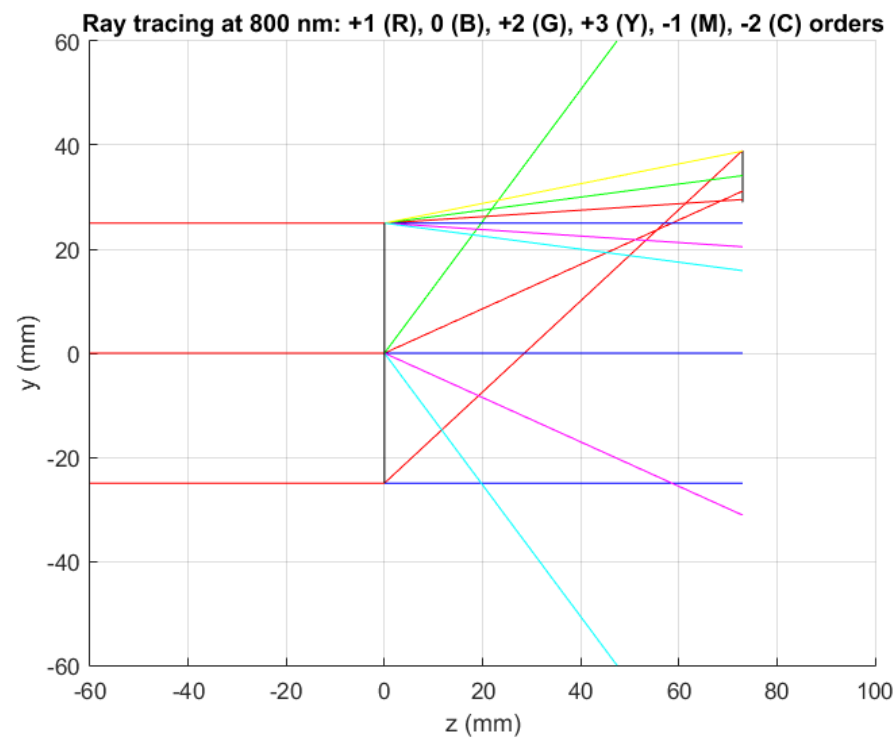

Figure 7. Ray tracing of rays corresponding to all diffraction orders $(-2,-1,0,+1,+2$ and +3 orders, depicted with cyan, magenta, blue, red, green and yellow lines, respectively) observed at the considered lens output when reconstructing with $800 \mathrm{~nm}$ and $\theta=0^{\circ}$. The black line at $z=73 \mathrm{~mm}$ represents a possible placement of the PV cell, where all $800 \mathrm{~nm}$ rays corresponding to the +1 diffracted order are collected. 
Simulations of the energetic performance of the lens and different placements of the $10 \mathrm{~mm}$ wide photovoltaic cell have been carried out. Solar movement tracking in the direction of highest angular selectivity is assumed, so the reconstruction angle is $\theta_{0}=0^{\circ}$, and the incident irradiance corresponds to the standard AM 1.5D solar spectrum normalized to $1000 \mathrm{~W} / \mathrm{m}^{2}$ over the range $350-1200 \mathrm{~nm}$. Fresnel reflection losses ${ }^{17}$ at the entrance and output interfaces have been taken into account. One of the optimum placements is the one depicted in Figure 7 , collecting all +1 order of diffraction rays of $800 \mathrm{~nm}$. The spectral irradiance received by the whole surface of the PV cell due to all diffraction orders is shown in Figure 8. As expected, the maximal spectral irradiance is concentrated around $800 \mathrm{~nm}$, and although this curve reaches values of irradiance lower than the ones obtained with the volume HOEs studied in the previous section, it is wider, so the total area under the curve is bigger and therefore, more energy is collected. Besides, the +2 order of diffraction rays with wavelengths around $450 \mathrm{~nm}$, and to a lesser extent the +3 order of diffraction, despite having lower irradiance, also reach the PV cell and contribute to the generation of current intensity.

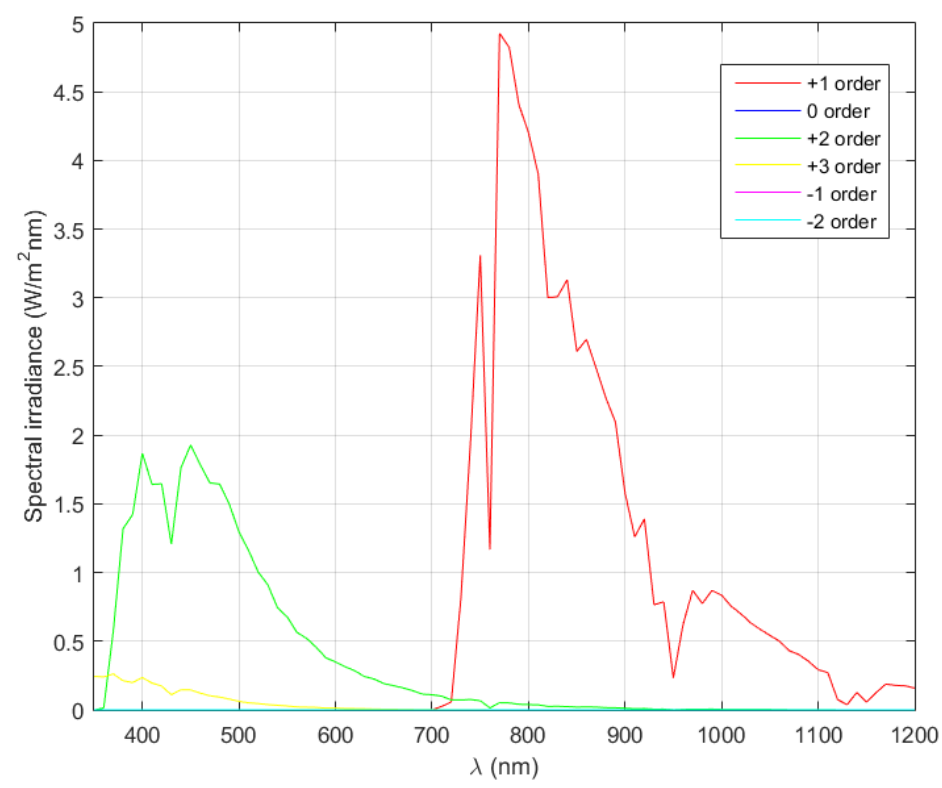

Figure 8. Spectral irradiance received by the PV cell placed according to Figure 7, with incoming standard AM 1.5D solar spectrum normalized to $1000 \mathrm{~W} / \mathrm{m}^{2}$ over the range 350-1200 nm and $\theta=0^{\circ}$, due to all orders of diffraction $(-2,-1,0,+1,+2$ and +3 orders, depicted with cyan, magenta, blue, red, green and yellow lines, respectively).

A total optical concentration coefficient (defined with equation (5)) of 1.01 was achieved, which is higher than the ones obtained in the previous section. It is roughly the same value that would be obtained with no optical concentration system, however, it has to be remembered that the concentrated spectral range corresponds mainly to the optimal wavelength range of the PV cell, so the generated current intensity would be higher than with no concentration system. In fact, if the spectral response of the cell is taken into account, a total current intensity concentration coefficient (defined with equation (6)) of 1.14 is obtained, which is a promising result.

Other factors also need to be noted. The spectral irradiance from the largest considered wavelengths that reaches the cell is not significantly higher than the corresponding values of the solar spectrum. That means that the temperature of the cell due to solar radiation will not be increased because of it.

Furthermore, similarly to the discussion on the previous section, this geometry allows the use of more than one holographic concentrator leading sun rays to the same PV cell, so the total irradiance received in the optimum spectral range and the generated current intensity would increase. 
Higher orders of diffraction need to be taken into account in the calculations when the HOE operates in the transition regime, because if not, the efficiency of the +1 diffracted order would be overestimated. Besides, depending on the placement of the PV cell, the rays corresponding to higher orders of diffraction might reach it as well and contribute to the generation of electricity and also to its increase of temperature.

\section{CONCLUSION}

Some energetic aspects of the behavior of holographic cylindrical lenses have been studied in this work by means of a developed ray tracing algorithm. On the one hand, a theoretical analysis of the effect of the recording wavelength on the efficiency of the diffracted beam has proved that it is not critical for this application, since the image quality of the reconstruction wave is not relevant. On the other hand, a holographic cylindrical lens operating both in the volume regime and the transition regime has been recorded, and the measured efficiency of every diffracted order that emerges is in good agreement with the calculated values obtained with the algorithm. The spectral irradiance that a PV cell at a certain location would collect has been calculated, providing higher concentration coefficients than the volume holographic cylindrical lenses considered. This shows that HOEs operating in the transition regime should be considered for solar concentration applications, where a broadband diffracted spectrum is desired.

Future work includes further analysis of holographic cylindrical lenses operating in the transition regime. A more thorough analysis of the coupling between the concentrating holographic system and the PV cell is also needed, in which the whole solar spectrum is considered, and not only the spectral range 350-1200 nm, in order to study the direction and energy of the rays with higher wavelengths. Applying a model of the behavior of the PV cell, an estimation of the cell temperature and the corresponding decrease of the current intensity can be made.

\section{AKNOWLEDGEMENTS}

This research was supported by the Ministerio de Economía y Competitividad of Spain with funding (grant ENE201348325-R), the Generalitat de Catalunya (grant 2016 FI_B1 00019) and the Diputación General de Aragón-Fondo Social Europeo (TOL research group, T76).

\section{REFERENCES}

[1] Collados, M. V., Chemisana, D., Atencia, J., "Holographic solar energy systems: The role of optical elements," Renew. Sustain. Energy Rev. 59, 130-140 (2016).

[2] Chemisana, D., Collados, M. V., Quintanilla, M., Atencia, J., "Holographic lenses for building integrated concentrating photovoltaics,” Appl. Energy 110, 227-235(2013).

[3] Bañares-Palacios, P., Álvarez-Álvarez, S., Marín-Sáez, J., Collados, M.-V., Chemisana, D., Atencia, J., "Broadband behavior of transmission volume holographic optical elements for solar concentration,” Opt. Express 23(11), A671-A681(2015).

[4] Fernández, R., Gallego, S., Márquez, A., Francés, J., Navarro-Fuster, V., Pascual, I., "Diffractive lenses recorded in absorbent photopolymers,” Opt. Express 24(2), 1559 (2016).

[5] Sam, S. T. L., Kumar, A. P. T., Predeep, P., Thakur, M., Varma, M. K. R., "Design and Optimization of Photopolymer Based Holographic Solar Concentrators,” Opt. Phenomena, Mater. Devices Charact. (AIP Conf. Proceedings), 248-250 (2011).

[6] Bianco, G., Ferrara, M. A., Borbone, F., Roviello, A., Pagliarulo, V., Grilli, S., Ferraro, P., Striano, V., Coppola, G., "Multiplexed holographic lenses : realization and optical characterization," 2015 Fotonica AEIT Ital. Conf. Photonics Technol., Institution of Engineering and Technology (2015).

[7] Akbari, H., Naydenova, I., Martin, S., "Using acrylamide-based photopolymers for fabrication of holographic optical elements in solar energy applications,” Appl. Opt. 53(7), 1343(2014). 
[8] Berneth, H., Bruder, F.-K., Fäcke, T., Jurbergs, D., Hagen, R., Hönel, D., Rölle, T., Walze, G., “Bayfol HX photopolymer for full-color transmission volume Bragg gratings,” Proc. SPIE. 9006, 900602 (2014).

[9] Marín-Sáez, J., Atencia, J., Chemisana, D., Collados, M.-V., "Characterization of volume holographic optical elements recorded in Bayfol HX photopolymer for solar photovoltaic applications,” Opt. Express 24(6), A720 (2016).

[10] Gordon, M., Zhang, D., Vorndran, S., Russo, J. M., Luscombe, C. K., Shaheen, S. E., Kostuk, R. K., "Planar holographic spectrum-splitting PV module design,” Proc. SPIE, 846808 (2012).

[11] Ludman, J. E., Riccobono, J., Reinhand, N. O., Semenova, I. V., Martín, J., Tai, W., Li, X., Syphers, G., "Holographic solar concentrator for terrestrial photovoltaics,” Proc. 24th IEEE Photovolt. Spec. Conf., 12081211 (1994).

[12] Ludman, J. E., Riccobono, J., Semenova, I. V., Reinhand, N. O., Tai, W., Li, X., Syphers, G., Rallis, E., Sliker, G., et al., "The optimization of a holographic system for solar power generation," Sol. Energy 60(1), 1-9, Elsevier Science Ltd (1997).

[13] Marín-Sáez, J., Chemisana, D., Moreno, Á., Riverola, A., Atencia, J., Collados, M.-V., “Energy Simulation of a Holographic PVT Concentrating System for Building Integration Applications,” Energies 9(8), 577 (2016).

[14] Marín-Sáez, J., Collados, M. V., Atencia, J., Chemisana, D., “Optical and Energetic Performance of Volume Holographic Optical Elements for Solar Energy Applications.” (in press).

[15] Kogelnik, H., “Coupled wave theory for thick hologram gratings,” Bell Syst. Tech. J. 48(9), 2909-2947 (1969).

[16] Syms, R., Practical Volume Holography, Oxford University Press (1990).

[17] Hecht, E., Optics, Addison Wesley (1998). 